Master of Science Thesis

\title{
ROBUST AND ACCURATE DIAPHRAGM BORDER DETECTION IN CARDIAC X-RAY ANGIOGRAPHIES
}

\author{
Simeon Petkov
}

Advisors: Carlo Gatta and Petia Radeva

Juny 2012 


\begin{abstract}
X-ray angiography is the most common imaging modality employed in the diagnosis of coronary diseases prior or during a catheter-based intervention. The analysis of the patient X-Ray sequence can provide useful information about the degree of arterial stenosis, the myocardial perfusion and other clinical parameters. If the sequence has been acquired to evaluate the perfusion grade, the opacity due to the diaphragm could potentially hinder any kind of visual inspection and make more difficult a computer aided measurements. In this thesis we propose an accurate and robust method to automatically identify the diaphragm border in each frame. Quantitative evaluation on a set of 11 sequences shows that the proposed algorithm outperforms previous methods.
\end{abstract}


I hope that this will help and will be used for something good. 


\section{Acknowledgements}

\section{Thanks to:}

Maria Nisheva from Sofia University Bulgaria. (I would enjoy it more if all the teachers are like you.)

Gennady Agre from Bulgarian Academy of Science. (I don't know what happened to that beer...)

Petia Radeva from Universitat de Barcelona. (I will always cherish the moment I got up the stairs in UB and met you.)

Carlo Gatta from Centre de Visió per Computador. (For guiding me to the left and to the right and giving me time to swing.)

Xavi from University Hospital 'Germans Trias i Pujol'. (For the assistance that I hope will credit more and more.)

Adriana from Universitat de Barcelona and Centre de Visió per Computador. (For the Insanity and not only for that.)

Stilian. (R.I.P.) (for making me motivated on getting some diploma - something that my mother had not succeeded before.)

All the persons that helped me, all my friends and all my enemies.

And of course - my mother Liliana. 


\section{Contents}

List of Figures vii

$\begin{array}{ll}\text { List of Tables } & \text { ix }\end{array}$

1 Introduction 1

2 Related Works 3

3 Method 5

3.1 Artery removal . . . . . . . . . . . . . . . . . 6

3.2 Edgeness . . . . . . . . . . . . . . . . . . . 6

3.3 From Edgeness to paths . . . . . . . . . . . . . . 8

3.4 Diaphragm border surface as a collection of paths . . . . . . . . . . 10

3.5 Imposing diaphragm border smoothness on image plane . . . . . . . . . 12

4 Validation $\quad 13$

4.1 Validation framework . . . . . . . . . . . . . . . . . 13

4.2 Results . . . . . . . . . . . . . . . . . . . . . 14

$\begin{array}{lll}5 & \text { Discussion } & 17\end{array}$

6 Conclusions $\quad 19$

$\begin{array}{ll}\text { References } & 21\end{array}$ 


\section{List of Figures}

3.1 Effect of the closing operator on the Edgeness result:

(a) input frame;

(b) after applying the morphological closing;

(c) the Edgeness measure applied on frame in (a); (d) the Edgeness measure applied on filtered frame in (b)

3.2 Average distances over the training set when varying $\sigma_{M} \ldots \ldots$. . . . . 9

3.3 Paths construction: (a) an example of paths construction for a given $x$; (b) Detail of the bottom left plot in (a); (c) A 3D plot of all the paths, where darker paths correspond to higher quality paths. . . . . . . . . . . 10

3.4 (a) The paths of Fig. 3.3(c) colored in accordance to their clusters; the green cluster is the selected one. (b) The resulting surface after fitting the polynomial function to all the sequence frames. . . . . . . . . . . . . 11

3.5 An example of iterative fitting of a polynomial function to the clustered points . . . . . . . . . . . . . . . . . . . . 12

4.1 Four results. Ground truth is marked with blue, the prediction of the method from [1] is red and our prediction is green. . . . . . . . . . . . 1 


\section{List of Tables}

4.1 Quantitative results. All measures are in pixels . . . . . . . . . . . . 16 


\section{1}

\section{Introduction}

When a human suffers from a heart attack or has a severe arterial stenosis the first thing medical doctors do is to restore the normal blood flow. To perform a diagnosis they insert a catheter into the main coronary arteries. Right after a successful restoration of normal artery blood flow a second important step is performed - estimating the myocardium healthiness. This is needed because during the time in which there was not enough blood supply to the heart, the heart tissue (myocardium) suffers and could slowly die. In order to examine this doctors inject an opaque liquid (called contrast liquid) through the catheter. The process is recorded as a video by means of a X-Ray machine. The video is then examined by a specialist to see if the dark liquid is well absorbed by the myocardium, which corresponds to healthy heart.

The Cardiac X-Ray angiography (Fluoroscopy) is the imaging modality widely used in the analysis of cardiac diseases prior or during catheterization interventions. Besides the estimation of stenosis degree, other qualitative and quantitative analysis can be performed: in the last five years, some semi-automatic tools for the quantitative computer assisted measurement of the myocardial perfusion level (known as MBG or TIMI-MPG) have been proposed $[2 ; 3 ; 4 ; 5]$. All of these methods are negatively affected by the diaphragm motion. In [2], authors make explicit use of a method for diaphragm border detection [1] to improve the quality of the region-of-interest tracking that is used to measure the myocardial perfusion. In [3], authors claim that the breathing movements can hide staining patterns, showing that the diaphragm movement, and the consequent gray-level variation in an area, can reduce the method ability to measure the myocardial staining. In [4], authors impose the angiography sequence acquisition to be done while 


\section{INTRODUCTION}

the patient holds breath and show that the diaphragm movement introduces artifacts in the resulting analysis. It has to be noted that not all the patients can hold breath for the time required to a complete myocardial perfusion analysis sequence. In the preliminary work in [5], authors claim that one limitation of their method is that the manually delineated perfusion area must be isolated from the diaphragm, so that the method applicability is reduced for certain angiographic projections. All these methods can benefit of a pre-processing step able to accurately detect and digitally remove the diaphragm border. Moreover, in the future, the digital removal of the diaphragm can be used as a tool to enhance visualization during catheter-based interventions.

The main contributions of our thesis are:

(1) A method which outperforms the state of the art, exploiting the diaphragm appearance, motion and morphological properties in a better way.

(2) The proposal of a publicly available dataset for a quantitative performance evaluation. 


\section{2}

\section{Related Works}

To the best of our knowledge, only one method for automatic detection of the diaphragm border has been proposed so far [1]. Authors model the diaphragm border as an arc of a circle. Their method is based on the following pipeline:

\section{Pre-processing the frames}

In their method, the authors first preprocess each frame from the X-Ray sequence, using a morphological closing operator to remove arteries. However, they do not define the shape and the size of the operator's structuring element, they only say that the size is slightly larger than the largest expected vessel. We follow the same approach for eliminating the arteries putting additional theoretical background when defining the operator's parameters.

2. Apply Canny edge detector

An edge detector is applied on each preprocessed frame and the assumption is that the diaphragm edge will somehow dominate. However the morphological closing operator always produces noise (introduces new edges) when removing the arteries and it is not elaborated further on how they handle that noise.

3. Use the Hough transform to detect circles

The next step in the method is to use Hough Transform [6] and perform extensive search in the circle parameters domain. The circle that best fits the highlighted edges is supposed to be the one that fits the diaphragm border best. 


\section{RELATED WORKS}

4. Apply an active contour model (snake) to refine the result

Since the diaphragm border may not have the shape of a circle arc, the final step of their method is to refine the border contour if a fitting criteria measure is not satisfactory enough. The refining is performed by a snake algorithm, optimizing a function that the authors do not define in their paper. 


\section{3}

\section{Method}

The proposed method has been developed exploiting the characteristics of the diaphragm in both spatial and temporal dimensions. We define a set of assumptions that will be used to obtain a robust and accurate diaphragm detection:

(1) The diaphragm border appears as a vertical transition (edge) from brighter (above) to darker (below) gray-scale levels;

(2) The diaphragm movement is continuous;

(3) The diaphragm has a moving pattern which differs from the cardiac motion pattern and that pattern could significantly change from patient to patient;

(4) The diaphragm border is a continuous smooth curve in the image domain;

These assumptions have been exploited in an algorithm whose steps can be summarized as follows:

(1) Roughly remove the arteries by means of a morphological closing operator;

(2) Compute an Edgeness measure based on vertical multi-scale gradients;

(3) Extract a set of paths traversing high (and at the same time similar) Edgeness values through the temporal axis;

(4) Perform an unsupervised clustering to determine the set of paths that composes the diaphragm; 
(5) For each frame, interpolate the optimal diaphragm shape while removing outlier paths.

\subsection{Artery removal}

As claimed in [1], the arterial staining can disturb the identification of the diaphragm border leading to vertical edges that locally resemble the diaphragm border appearance. Moreover, edges caused by arteries are normally stronger than the ones due to the diaphragm border. To deal with this problem, we apply a morphological closing operator as in [1], using as structuring element a disk of radius $R$ pixels on each sequence frame separately. Having an average resolution of $0.34 \mathrm{~mm} /$ pixel, to roughly remove arteries up to a diameter of $6 \mathrm{~mm}$, the optimal radius is $R=6[\mathrm{~mm}] / 0.34[\mathrm{~mm} / \mathrm{pixel}]=$ $20[$ pixel]; this is sufficient to remove even thick left main arteries [7]. Fig. 3.1 (b) shows an example of the closing filtering. It can be noticed that the removal is not very accurate; however this is not critical since the diaphragm border is maintained and successive steps of our algorithm allow to delineate it robustly.

\subsection{Edgeness}

Let us define a gray-level sequence as a volume where two coordinates correspond to the image plane and the third coordinate is time, so that we can write it as a function $I(x, y, t) \in \mathbb{R}$. With the aim of exploiting the first assumption, we compute the normalized vertical derivative of all sequence frames at different scales as follows:

$$
V_{\sigma}(x, y, t)=\sigma I(x, y, t) * \frac{\partial G(0 ; \sigma)}{\partial y}
$$

where the symbol $*$ denotes the convolution operator and $G$ is a Gaussian kernel with zero mean and standard deviation $\sigma$. We use the Lindeberg normalization [8] so that derivatives at different scales are comparable. Since we are searching for edges where the upper part is brighter than the lower part, we can modify $V_{\sigma}(x, y, t)$ to cancel its values for edges with the opposite pattern as follows:

$$
\tilde{V}_{\sigma}(x, y, t)=\left\{\begin{array}{ccc}
V_{\sigma}(x, y, t) & \text { if } & V_{\sigma}(x, y, t)>0 \\
0 & \text { if } & V_{\sigma}(x, y, t) \leq 0 .
\end{array}\right.
$$




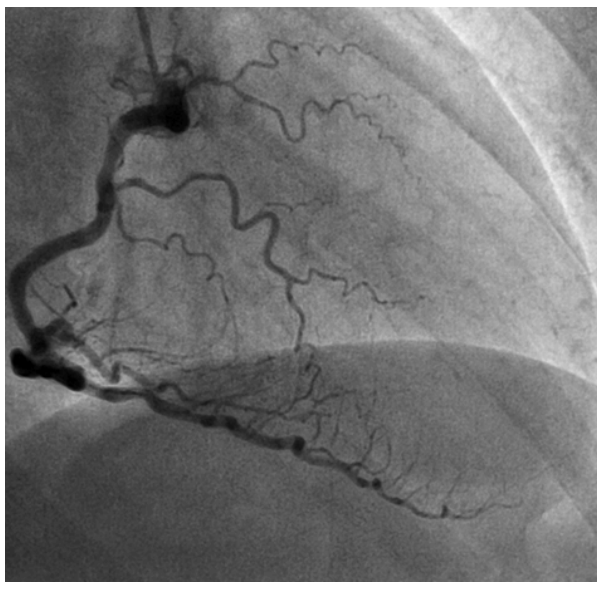

(a)

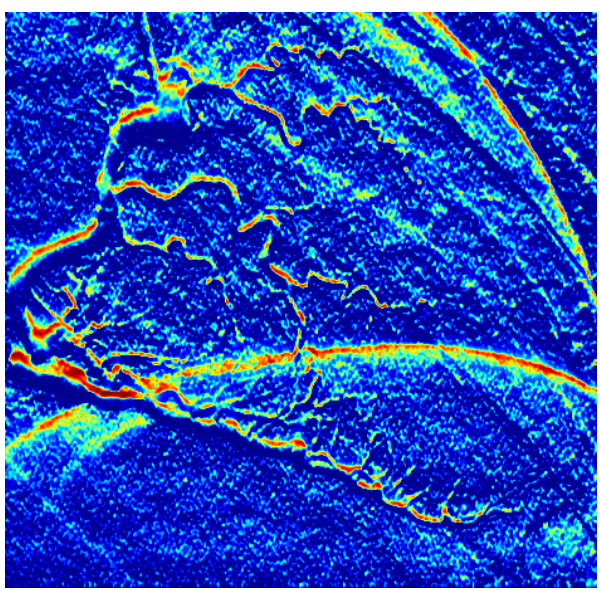

(c)

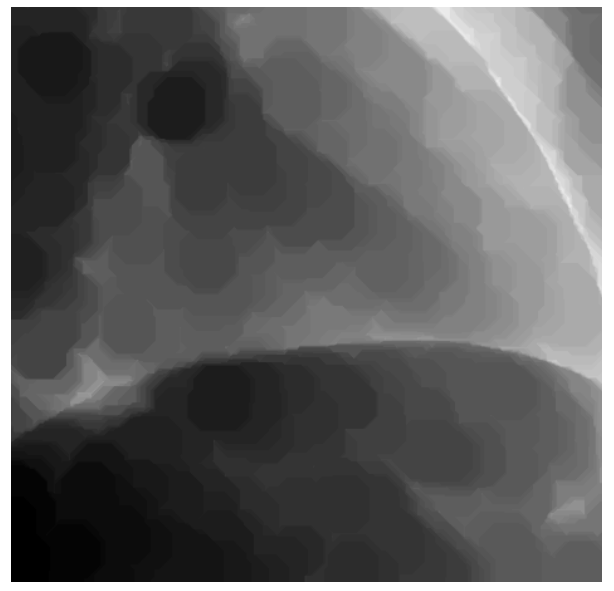

(b)

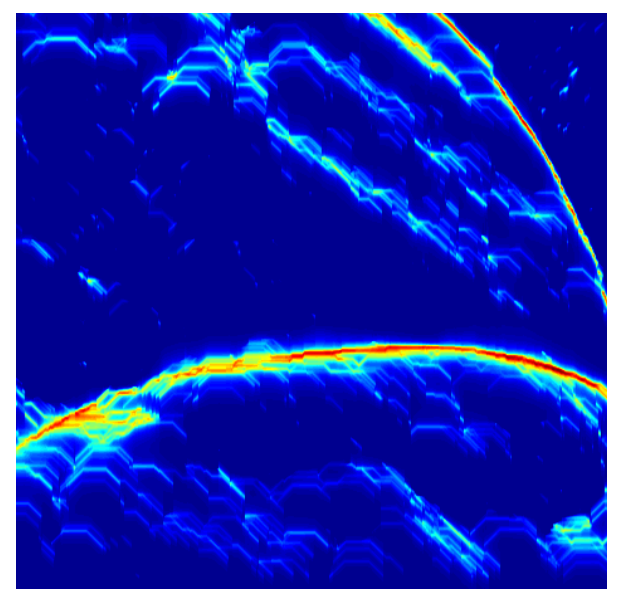

(d)

Figure 3.1: Effect of the closing operator on the Edgeness result:

(a) input frame;

(b) after applying the morphological closing;

(c) the Edgeness measure applied on frame in (a);

(d) the Edgeness measure applied on filtered frame in (b). 


\section{METHOD}

To discriminate between noisy edges and consistent edges over scales, we compute the average vertical modified Edgeness as follows:

$$
\tilde{E}(x, y, t)=\frac{1}{|\Phi|} \sum_{\sigma \in \Phi} \tilde{V}_{\sigma}(x, y, t)
$$

where the set of scales is $\Phi=\{1,2,4,8,16\}$ pixels. The scales have been defined in octaves to cover all possible sizes of edges caused by the diaphragm border; values larger than 16 do not provide relevant information and would merely increase the computational cost of the algorithm. Moreover, thanks to the averaging process, only clear edges will have large positive values of $E$. We apply the non-linear transformation to the Edgeness map:

$$
E(x, y, t)=1-\exp (-\tilde{E}(x, y, t))
$$

$E(x, y, t) \in[01]$ and has higher values at edges resembling a diaphragm border. Fig. 3.1(c-d) shows two examples of Edgeness map respectively for the frames (a-b) - it can be seen that the closing operator performs a sufficient artery removal.

\subsection{From Edgeness to paths}

Let us denote a path $p$ as a curve with a fixed $x$ coordinate and a $y$ coordinate varying as a function of time: $p=\left\{x_{p}, y_{p}(t)\right\}$. From the first frame, we select all pixels having a value $E(x, y, 1)>\mu(E)$, i.e. above the average Edgeness for the sequence and use them as starting points. For each starting point from the first frame $(t=1)$, we draw a path by vertically tracking that point from frame to frame (sequentially from $t=2$ to the end of the sequence $t=T$ ). The tracking maximizes the path quality defined with the following formula:

$$
Q_{\mathrm{path}}(p)=\frac{1}{T} \sum_{t=1}^{T} E\left(x_{p}, y_{p}(t), t\right) \cdot S\left(y_{p}(t), y_{p}(t-1)\right) \cdot M\left(y_{p}(t), \tilde{y}_{p}(t-1)\right)
$$

This formula can be split in three parts, which account for different aspects. The rational behind the first term $E(\cdot, \cdot, \cdot)$ is to encourage the path to transverse points with high Edgeness values. However, this alone can lead to scattered paths jumping from one maxima to another in the next frame. The second (similarity) term

$$
S\left(y_{p}(t), y_{p}(t-1)\right)=1-\left|E\left(x_{p}, y_{p}(t), t\right)-E\left(x_{p}, y_{p}(t-1), t-1\right)\right| \in[01]
$$


imposes minimal Edgeness value variation between consecutive path points. The third term

$$
M\left(y_{p}(t), \tilde{y}_{p}(t)\right)=e^{-\frac{\left(\tilde{y}_{p}(t)-y_{p}(t)\right)^{2}}{2 \sigma_{M}^{2}}}
$$

has been designed to add a smoothness criterion to the path construction. Here $\tilde{y}_{p}(t)$ is the expected value for the time location $t$, based on the linear approximation of previous values $^{1}$ :

$$
\tilde{y}_{p}(t)=2 y_{p}(t-1)-y_{p}(t-2)
$$

Basically, the use of a Gaussian (with its $\sigma_{M}$ parameter) centered around the expected location $\tilde{y}_{p}(t)$ adds a constraint, so that paths are restricted from changing their trajectories in a way that does not resemble a continuous movement. All of the three terms are bounded in $[01]$, so that their product ensures that only points fulfilling the three criteria at once are selected. The smoothness parameter $\sigma_{M}$ has been defined by crossvalidation. Fig. 3.2 shows the plotted error for different $\sigma_{M}$ values using a training set of 9 images. As it can be seen $\sigma_{M}=5$ [pixels] is the optimal choice. Changing the linear model for predicting $\tilde{y}_{p}(t)$ with a second order degree polynomial resulted in lower performance. Since the proposed path building is based on local decisions maximizing

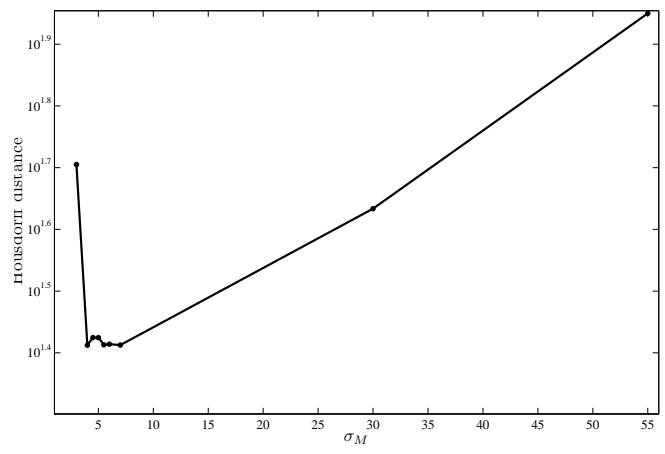

Hausdorff distance

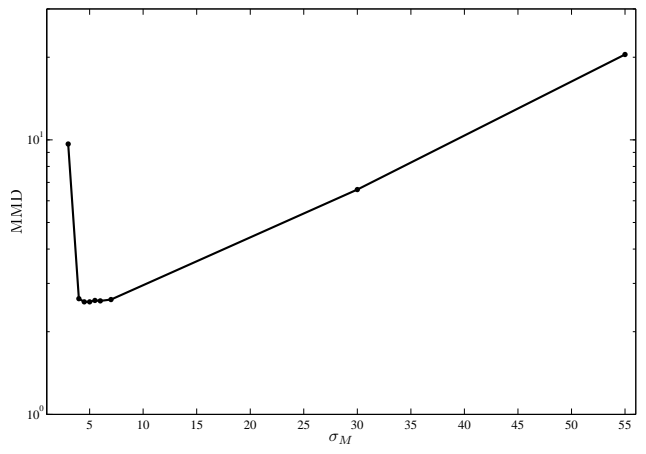

MMD

Figure 3.2: Average distances over the training set when varying $\sigma_{M}$.

equation (3.1), we allow the construction of multiple paths given an $x$. However, to avoid path proliferation, if two paths collide, only the one with higher path quality is retained, so that at the end of the process we have a set of non-intersecting continuous

\footnotetext{
${ }^{1}$ We make the 'zeroth' and the 'minus first' frames the same as the first one.
} 


\section{METHOD}

paths $\mathcal{P}$. Fig. 3.3(c) depicts an example of resulting paths. Fig. 3.3(a) shows an example of paths construction over-imposed on the values of $E(x, y, t)$ for a specific $x$. As it can be noticed, the paths are smooth while accurately following the local shape of $E(x, y, t)$. Moreover, the paths quickly collapse to a limited number of paths (see Fig. $3.3(\mathrm{~b}))$.

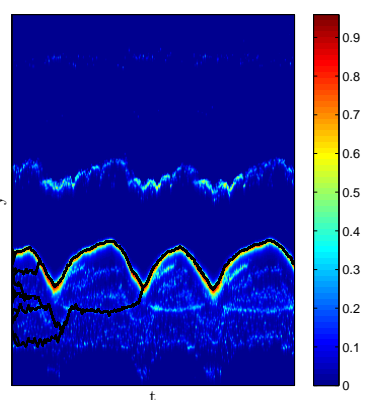

(a)

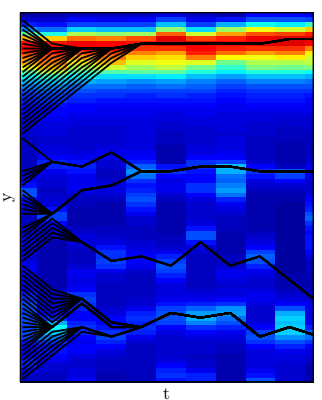

(b)

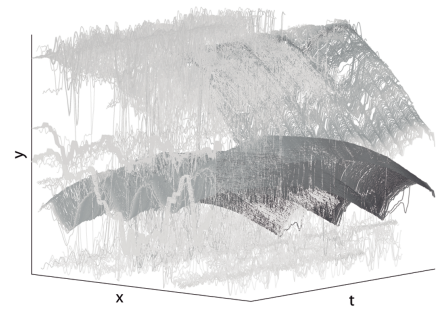

(c)

Figure 3.3: Paths construction:

(a) an example of paths construction for a given $x$;

(b) Detail of the bottom left plot in (a);

(c) A 3D plot of all the paths, where darker paths correspond to higher quality paths.

\subsection{Diaphragm border surface as a collection of paths}

The previous step generates a set of paths $\mathcal{P}$. Each path $p$ has its own trajectory $y_{p}(t)$ and a quality measure $Q_{\text {path }}(p)$. We want to select a subset $\mathcal{D} \subseteq \mathcal{P}$ which contains paths that move in a similar way, so that we make use of the assumption that the diaphragm has a specific motion pattern. Moreover, we want to retain high quality paths and discard low quality paths, which normally correspond to false positive detections. To accomplish these two steps in a unified way, we perform an unsupervised clustering of both motion characteristics and quality measure: (1) Each path is modified so that we remove its bias, $\hat{y}_{p}(t)=y_{p}(t)-\mu\left(y_{p}(t)\right)$, where $\mu$ is the mean operator. This step removes the average vertical position of the paths so that the clustering will not be influenced by (vertical) proximity of paths. (2) A feature vector $\vec{v}_{p}=\left[\hat{y}_{p}(1) \ldots \hat{y}_{p}(T) Q_{\text {path }}(p)\right]$ made of $T+1$ elements is built. This vector contains information on path motion (first $T$ elements) and information on the path quality (last element). (3) We build 
a matrix $\vec{V}$ where each row contains a feature vector $\vec{v}_{p}$ for each path in $\mathcal{P}$. Since vertical positions and quality measure are incommensurable we need to normalize $\vec{V}$ such that every column has zero mean and unitary standard deviation. (4) We apply an unsupervised k-means* clustering; the cluster with the highest mean quality is the one which most probably defines the diaphragm border as a collection of paths. The use of unsupervised clustering is the most suited way to group paths into a consistent result since the actual diaphragm motion is unpredictable: a sequence can be acquired while the patient is holding the breath, or the patient can have irregular breathing pattern. On the other way, k-means requires the definition of the number of clusters, which we set to three. The rational behind this setting is to allow the clustering to find one cluster for the diaphragm, one cluster for noisy low quality paths and a third cluster which has a motion pattern that is a mixture of the diaphragm and cardiac patterns. This latter case has been considered since the morphological operator is not able to eliminate non-tubular structures that move according to the cardiac cycle. Fig. 3.4(a) shows the result of clustering for the case in Fig. 3.3(c); different clusters are depicted in different colors.

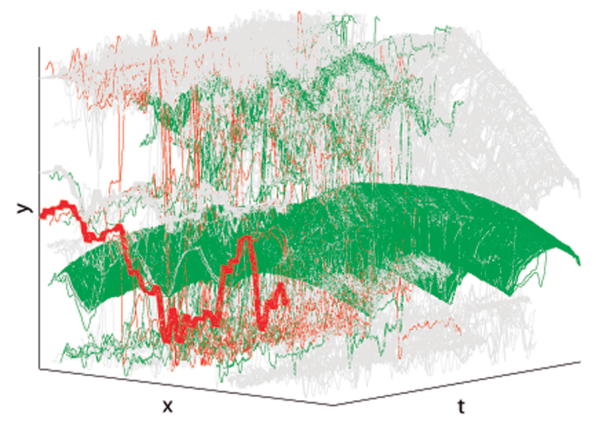

(a)

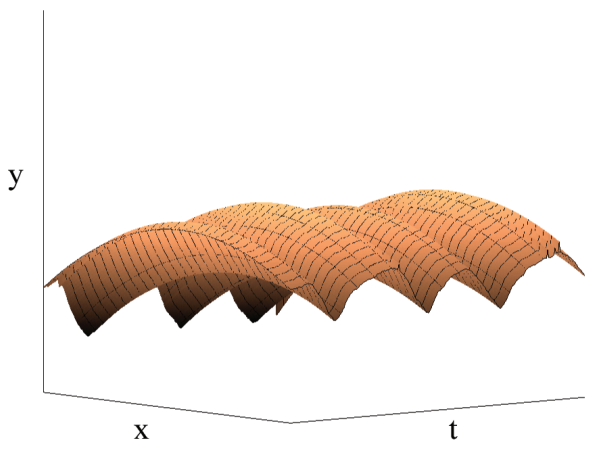

(b)

Figure 3.4: (a) The paths of Fig. 3.3(c) colored in accordance to their clusters; the green cluster is the selected one. (b) The resulting surface after fitting the polynomial function to all the sequence frames.

* Since the k-means is based on random centroids initialization, and it is not computational costly, we perform 100 trials keeping the solution with the lowest objective function. 


\subsection{Imposing diaphragm border smoothness on image plane}

The previous step defines the diaphragm border evolving curve as a surface composed by a collection of paths having similar motion and high quality. Nonetheless, the clustering could be suboptimal and paths actually not belonging to the diaphragm border can fall into the set $\mathcal{D}$. Moreover, up to this point, no smoothness in the image plane has been imposed. The algorithm's last step is devoted to these two goals: imposing smoothness in the image plane while removing outlier paths. The diaphragm border is modeled as a polynomial function $f(x)=a x^{2}+b x+c$. To estimate the optimal triplet $\{\hat{a}, \hat{b}, \hat{c}\}$ for a given frame at time $t$, we employ the following iterative algorithm. We define a set of points $\mathcal{C} \triangleq\left\{\left(x_{i}, y_{i}\right)\right\}$ taken from the paths of the diaphragm cluster at time $t$. At each iteration the optimal parameters are estimated minimizing the fitting squared error Err $=\sum_{\left(x_{i}, y_{i}\right) \in \mathfrak{e}}\left(f\left(x_{i}\right)-y_{i}\right)^{2}$; If the Thompson Tau outliers detector [9] on the signed error distribution finds at least one outlier, the point $\left(x_{j}, y_{j}\right)=\arg \max _{i}\left|f\left(x_{i}\right)-y_{i}\right|$ is removed from the set $\mathcal{C}$ and the iterative algorithm continues. If no outliers are found, the algorithm stops. Fig. 3.5 shows an example of this algorithm while Fig. 3.4(b) shows the final surface result.

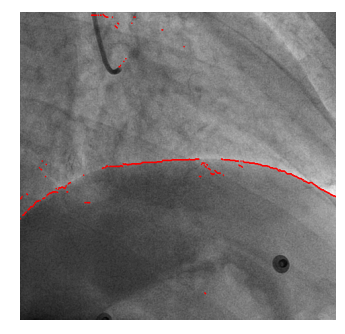

Initial points

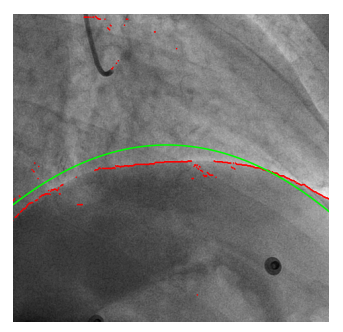

$1^{\text {th }}$ iteration

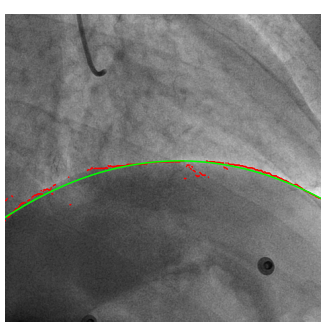

$50^{\text {th }}$ iteration

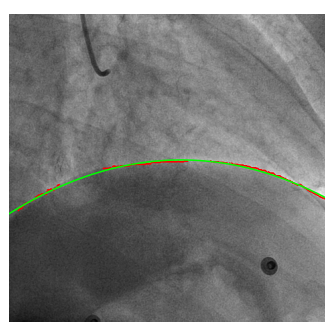

$155^{\text {th }}$ iteration (last)

Figure 3.5: An example of iterative fitting of a polynomial function to the clustered points. 


\section{4}

\section{Validation}

\subsection{Validation framework}

Material: We defined a validation set of 16 frames taken from 11 sequences (11 patients). The diaphragm border in each frame has been marked blindly by two experts. All sequences have been acquired using a Philips Allura Xper FD20, at 12.5 fps and with an image resolution of $0.34 \times 0.34 \mathrm{~mm}$. The $\mathrm{C}$-arm position varies from $-41^{\circ}$ to $97^{\circ}$ for the primary angle and from $-17^{\circ}$ to $33^{\circ}$ for the secondary angle.

Methods: We compare our proposed method with the diaphragm detection algorithm described in [1]. We also compute the inter-observer variability between the two ground truth annotations. Moreover, we also provide intermediate evaluations of algorithms.

Validation protocol: Let $G T$ be the set of points forming the ground truth curve and $P$ be the set of points predicted by a given method. We use two different measures to validate each prediction against the ground truths: (1) The Hausdorff distance [10] is the distance between the two most faraway points from the two sets of points. This measure is very sensitive to predicted points laying far from the ground truth points but gives little information about the overall precision of the prediction, thus making it useful in measuring robustness. (2) The Mean Minimal Distance

$$
M M D(P, G T)=\frac{1}{|P|} \sum_{i \in P} \min _{j \in G T}\left\|\left(x_{i}^{P}, y_{i}^{P}\right)-\left(x_{j}^{G T}, y_{j}^{G T}\right)\right\|
$$




\section{VALIDATION}

on the other hand, provides information about the overall precision of the predicted result. It has to be noted that the $M M D$ is not symmetric.

\subsection{Results}

Table 4.1 shows the error results using the Hausdorff and the MMD distances for the following cases (one for each row): inter-observer variability; the method in [1] before and after the application of the snake; our method prior to the clustering (Our-PC), prior to the curve fitting (Our-PF) and the final result (Our). For each distance, tables report its mean, standard deviation, minimum and maximum values.

An interesting fact is that the use of snakes, as expected, can help the method in [1] to improve the results but in a very limited extent. Regarding our method, it can be noticed that each step improves the results. Once the paths are built, before the clustering, the method is limited in the fact that the sequence is analyzed mainly in its temporal dimension, so that a large number of incorrect paths can be generated. The clustering step selects a set of paths that potentially represent the diaphragm border; this decreases consistently the MMD error (three times), but is not able to reduce the Hausdorff distance since erroneous paths far from the solution could be maintained. The polynomial fitting ensures a consistent solution imposing smoothness in the image domain and, at the same time, rejects erroneous paths thanks to the outlier detection; this results in a huge improvement in both the Hausdorff and MMD distances (more than one order of magnitude).

Results confirm that taking advantage of temporal and spatial properties of the diaphragm in a unified way produces far better results than relying only on frame based appearance. Fig. 4.1 shows four results:

Case (a) Both methods detect the diaphragm robustly, while our method (in green) shows a higher accuracy.

Case (b) A more difficult case - the method in [1] fails to detect a segment of the diaphragm border.

Cases (c) and (d) show that sometimes the diaphragm parabolic model might be too simple. 


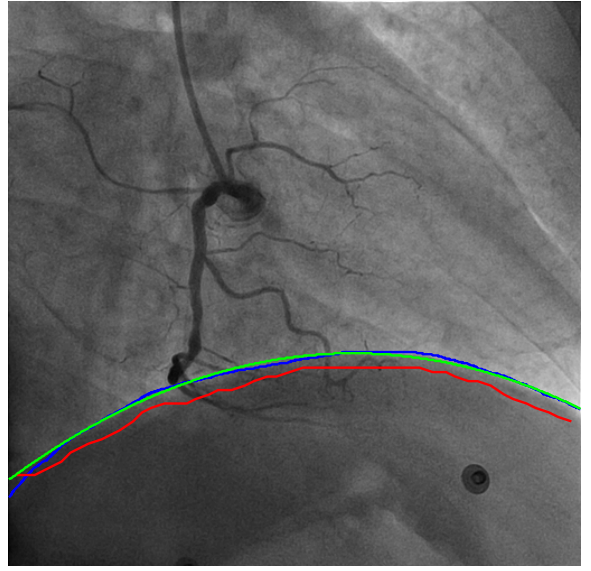

(a)

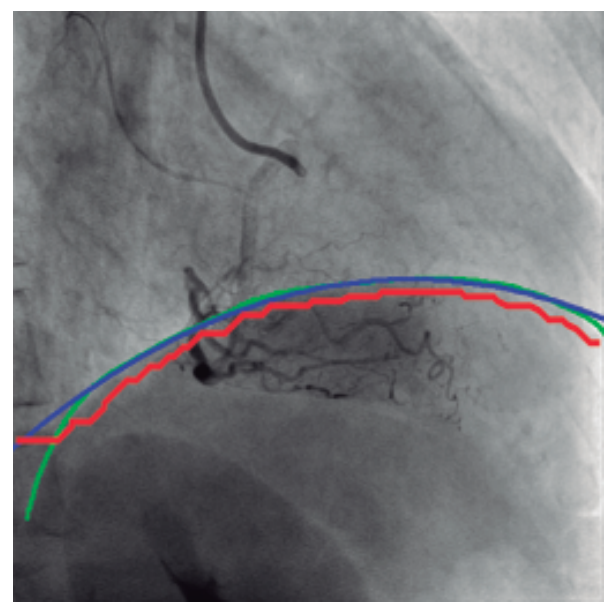

(c)

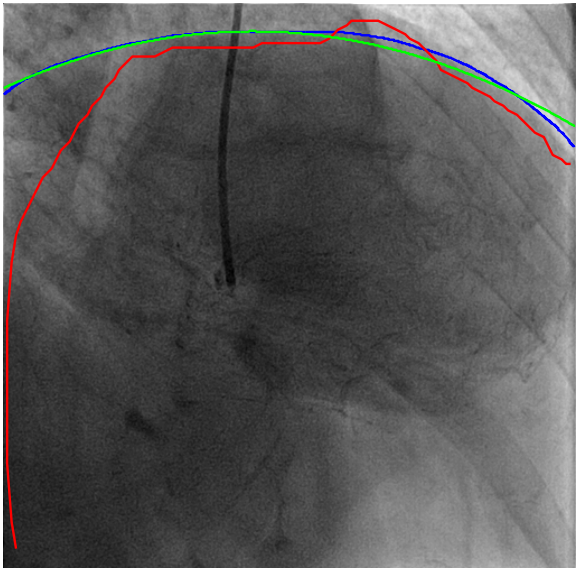

(b)

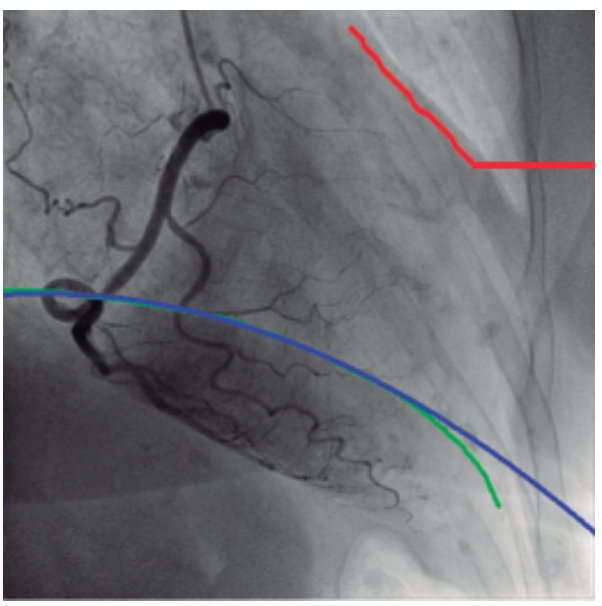

(d)

Figure 4.1: Four results. Ground truth is marked with blue, the prediction of the method from [1] is red and our prediction is green. 


\begin{tabular}{|c||c|c|c||c|c|c|}
\hline \multicolumn{1}{|c||}{} & \multicolumn{3}{c||}{ Hausdorff distance } & \multicolumn{3}{c|}{ MMD } \\
\hline & Avg \pm Std & $\min$ & $\max$ & Avg \pm Std & $\min$ & $\max$ \\
\hline \hline O1 vs O2 & $25.82 \pm 22.75$ & 4 & 86.33 & $2.03 \pm 2.07$ & 0.78 & 7.84 \\
\hline O2 vs O1 & $25.82 \pm 22.75$ & 4 & 86.33 & $1.41 \pm 0.66$ & 0.75 & 3.35 \\
\hline \hline$[1]$ (no snakes) & $83.36 \pm 124.37$ & 22.14 & 429.17 & $32.09 \pm 50.65$ & 14.24 & 233.49 \\
\hline$[1]$ & $84.71 \pm 120.73$ & 17.03 & 407.12 & $28.04 \pm 54.42$ & 10.71 & 234.17 \\
\hline \hline Our-PC & $364 \pm 48.5$ & 287 & 440 & $132 \pm 19.7$ & 108 & 185 \\
\hline Our-PF & $302 \pm 58.1$ & 188 & 385 & $43.7 \pm 25.5$ & 16.7 & 85.0 \\
\hline Our & $\mathbf{2 9 . 1} \pm \mathbf{2 3 . 5}$ & $\mathbf{5 . 4}$ & $\mathbf{9 7 . 2}$ & $\mathbf{3 . 0 0} \pm \mathbf{1 . 8 1}$ & $\mathbf{1 . 3 4}$ & $\mathbf{9 . 5 5}$ \\
\hline
\end{tabular}

Table 4.1: Quantitative results. All measures are in pixels 


\section{5}

\section{Discussion}

The proposed method shows some very good results, both in robustness and precision. While demonstrating potential it also reveals some of the challenging key points that still need to be solved before the ideas here reach trustful clinical practice.

Parabola fitting: It is not always the case that the diaphragm border has the shape of a parabola (fixed in rotation) - Fig. 4.1 (c) and (d). It could be that the polynomial model for the diaphragm border is too simple and that raises questions about balance between robustness and precision.

More than one diaphragm borders: It may be the case when the diaphragm borders are more than one. Biologically the diaphragms are two and in some projections both of them are present.

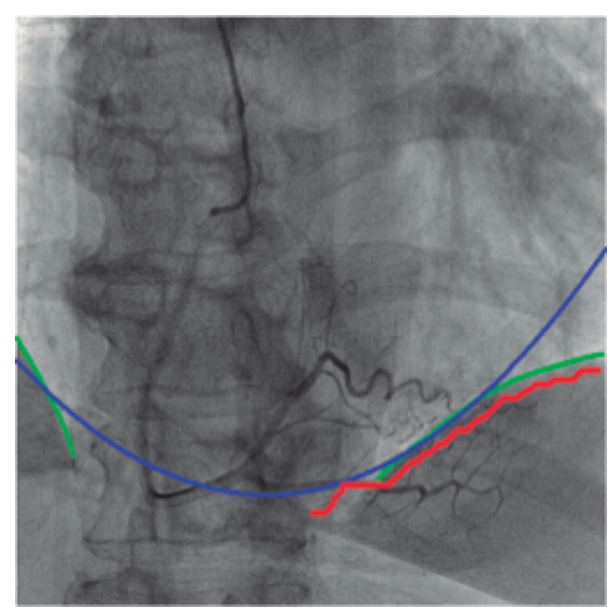


5. DISCUSSION 


\section{6}

\section{Conclusions}

In this thesis we proposed an algorithm for the robust and accurate detection of the diaphragm border in X-ray angiographies plus a validation methodology for quantitative performance evaluation. Results show that the proposed method is both robust and accurate. However, further investigation on how to deal with highly challenging cases is required. As future works, we want to:

(1) Increase the validation dataset

(2) Propose a digital diaphragm removal algorithm based on the detection proposed herein.

(3) Apply the method in an automatic tool for estimating the myocardial perfusion level.

The method described in this thesis has been submitted as a paper to the International Workshop on Statistical Atlases and Computational Models of the Heart, which is part of MICCAI 2012 conference. 
6. CONCLUSIONS 


\section{References}

[1] A. Condurache, T. Aach, K. Eck, J. Bredno, and T. Stehle, "Fast and robust diaphragm detection and tracking in cardiac x-ray projection images," in Spie MI, vol. 5747, pp. 1766-1775, 2005. vii, 1, 3, 6, 13, 14, 15, 16

[2] A. Condurache, T. Aach, A. Kaiser, and P. Radke, "User-defined ROI tracking of the myocardial blush grade," in 7th IEEE SSIAI, (Denver, CO), pp. 66-70, IEEE Computer Society, March 28-30 2006. 1

[3] D. Gil, O. Rodriguez-Leor, P. Radeva, and J. Mauri, "Myocardial perfusion characterization from contrast angiography spectral distribution," IEEE TMI, vol. 27, no. 5, pp. 641-649, 2008. 1

[4] J. Liénard and R. Vaillant, "Quantitative tool for the assessment of myocardial perfusion during x-ray angiographic procedures," in Proceedings of the 5th ICFIMH, FIMH '09, (Berlin, Heidelberg), pp. 124-133, Springer-Verlag, 2009. 1

[5] C. Gatta, J. D. G. Valencia, F. Ciompi, O. Rodriguez-Leor, and P. Radeva, "Toward robust myocardial blush grade estimation in contrast angiography," in IbPRIA, pp. 249-256, 2009. 1, 2

[6] R. Duda and P. Hart, "Use of the hough transformation to detect lines and curves in pictures," Tech. Rep. 36, AI Center, SRI International, 333 Ravenswood Ave, Menlo Park, CA 94025, Apr 1971. SRI Project 8259 Comm. ACM, Vol 15, No. 1. 3

[7] N. Funabashi, Y. Kobayashi, M. Perlroth, and G. Rubin, "Coronary artery: quantitative evaluation of normal diameter determined with electron-beam ct compared 
with cine coronary angiography initial experience.," Radiology, vol. 226, no. 1, pp. 263-71, 2003. 6

[8] T. Lindeberg, "Principle for automatic scale selection," tech. rep., RIT, 1998. 6

[9] T. R., "A note on restricted maximum likelihood estimation with an alternative outlier model," Journal of the Royal Statistical Society. Series B, vol. 47, pp. 5355, 1985. 12

[10] J. Henrikson, "Completeness and total boundedness of the hausdorff metric.," MIT Undergraduate Journal of Mathematics, vol. 1, pp. 69-80, 1999. 13 